\title{
Stability of Relative Equilibria in the Full Two-Body Problem
}

\author{
D.J. SCHEERES \\ The University of Michigan, Ann Arbor, Michigan, USA
}

\begin{abstract}
The stability of relative equilibrium solutions for the interaction of two massive bodies is explored. We restrict ourselves to the interaction between an ellipsoid and a sphere, both with finite mass. The study of this problem has application to modeling the relative dynamics of binary asteroids, the motion of spacecraft about small bodies, and the dynamics of gravity gradient satellites. The relative equilibrium can be parameterized by a few constants, including the mass ratio of the two bodies, the shape of the ellipsoid, and the normalized distance between the two bodies. Planar stability is characterized over this range of parameter values. When restricted to motion in the symmetry plane, the dynamical problem can be reduced to a two-degrees of freedom Hamiltonian system, which allows for an efficient computation of stability characteristics of the relative equilibria. Future work will look at full stability of these relative equilibria.
\end{abstract}

KEYWORDS: stability; relative equilibria; full two-body problem

\section{INTRODUCTION}

Our work uses the papers by Maciejewski ${ }^{1,2}$ as a take-off point for the current analysis. The important contributions by these papers include the use of body-fixed coordinates to describe the mutual dynamics of the two bodies, and the elimination of the angular momentum integral for a specialized, planar system. In the current work we look at the relative equilibria for an ellipsoid-sphere problem, and determine the planar stability of such a system. Although interesting, this work must eventually be expanded to consider the stability of the full non-planar problem.

This study is motivated by the observation that the stable configuration of a gravity gradient satellite aligns its axis of minimum inertia along the line connecting the two bodies. When the extended body is more massive than the sphere, such as for the motion of a spacecraft around an asteroid, it is easy to show that the same relative configuration between the bodies is unstable. Thus, there must be a stability bifurcation in the system as the mass ratio between the two bodies is shifted from the one extreme to the other. Closer examination of the stability bifurcation diagram for this system reveals a rich structure, and allows for direct comparison of the diagram with recent observations of binary asteroid systems.

Address for correspondence: D.J. Scheeres, The University of Michigan, Ann Arbor, MI 48109-2140, USA.

scheeres@umich.edu

Ann. N.Y. Acad. Sci. 1017: 81-94 (2004). @ 2004 New York Academy of Sciences. doi: 10.1196/annals.1311.006 


\section{FUNDAMENTALS}

\section{Mutual Potential}

The mutual gravitational potential between two arbitrary bodies can be written as follows:

$$
V=-\int_{\mathcal{B}_{1}} \int_{\mathcal{B}_{2}} \frac{G d m_{1} d m_{2}}{\left|\mathbf{r}_{1}-\mathbf{r}_{2}\right|},
$$

where $\mathcal{B}_{j}$ represents the $j$ th body, $d m_{i}$ represents a mass element of the $i$ th body, and $\boldsymbol{r}_{i}$ represents the position of the $i$ th mass element. Implicit in this formula are the relative positions and attitudes of the two bodies. Since one body is a sphere, a quadrature over the body can be immediately performed:

$$
V=-G M_{s} \int_{\mathcal{B}_{c}} \frac{d m_{c}}{\left|\mathbf{r}_{c}-\mathbf{r}_{s}\right|},
$$

where the subscript $s$ stands for the sphere, the subscript $c$ stands for the general body, and $\boldsymbol{r}_{c}$ is the position vector of the mass element $d m_{c}$ in the general body. $M_{S}$ denotes the total mass of the sphere and $\boldsymbol{r}_{s}$ denotes the position vector of the center of the sphere. This equation corresponds to the gravitational potential of a single arbitrary body and can be integrated and expressed in a variety of ways. We assume the quadrature takes place in a coordinate frame fixed in the general body at its center of mass. The mutual potential then becomes

$$
\begin{gathered}
V=-G M_{s} M_{c} \tilde{U}(\mathbf{R}) \\
\tilde{U}(\mathbf{R})=\frac{1}{M_{c}} \int_{\mathcal{B}_{c}} \frac{d m_{c}(\rho)}{|\mathbf{R}+\rho|},
\end{gathered}
$$

where $\mathbf{R}$ is the relative vector between the general body and the sphere and $\rho$ is the position vector of mass elements in the general body, both vectors expressed in the general-body-fixed frame. The function $\tilde{U}$ is the unit mass gravity potential of the general body.

The relative vector $\mathbf{R}$ can also be expressed in terms of the centers of mass of the two bodies:

$$
\begin{aligned}
\mathbf{R} & =\mathbf{A}^{T} \cdot \mathbf{r}_{I} \\
& =\mathbf{A}^{T} \cdot\left(\mathbf{r}_{s}-\mathbf{r}_{c}\right),
\end{aligned}
$$

where $\mathbf{A}$ is the attitude matrix of the general body (i.e., the transformation matrix that takes the general body frame to the inertial frame), $\mathbf{A}^{T}$ is the transformation matrix that goes from the inertial frame to the general body frame, $\mathbf{r}_{I}$ is the relative vector between the bodies in inertial space, and $\mathbf{r}_{i}$ is the inertial position vector of the $i$ th body. In this fully-coupled problem, the attitude matrix $\mathbf{A}$ is an implicit function of the general body and sphere positions, velocities, angular velocities, and time. The gravitational potential can be reduced to this simplified form because the absolute attitude of the sphere can be ignored (i.e., represented as an identity transformation), allowing the potential to be specified as a function of the relative positions of the centers of mass in the general-body-fixed frame. A more comprehensive review of the mutual gravitational potential can be found elsewhere. ${ }^{2}$ 


\section{Translational Equations of Motion}

The translational equations of motion of the two bodies in inertial space are

$$
\begin{aligned}
& \ddot{\mathbf{r}}_{s}=\frac{\partial V}{\partial \mathbf{r}_{s}} \\
& \ddot{\mathbf{r}}_{c}=\frac{\partial V}{\partial \mathbf{r}_{s}} .
\end{aligned}
$$

Replace the inertial position vectors with the center of mass vector $\mathbf{r}_{C M}$ and the relative vector $\mathbf{r}_{I}$, still specified in inertial space:

$$
\begin{gathered}
\mathbf{r}_{C M}=\frac{1}{M_{s}+M_{c}}\left(M_{s} \mathbf{r}_{s}+M_{c} \mathbf{r}_{c}\right) \\
\mathbf{r}_{I}=\mathbf{r}_{s}-\mathbf{r}_{c} .
\end{gathered}
$$

Now note that $\partial V / \partial \mathbf{r}_{I}=\partial V / \partial \mathbf{r}_{s}=\partial V / \partial \mathbf{r}_{c}$ to find

$$
\begin{gathered}
\ddot{\mathbf{r}}_{C M}=0 \\
\ddot{\mathbf{r}}_{I}=-\frac{M_{s}+M_{c}}{M_{s} M_{c}} \frac{\partial V}{\partial \mathbf{r}_{I}} \\
=-G\left(M_{s}+M_{c}\right) \frac{\partial \tilde{U}}{\partial \mathbf{r}_{I}} \\
=-\frac{\partial U}{\partial \mathbf{r}_{I}},
\end{gathered}
$$

where $U=G\left(M_{s}+M_{c}\right) \tilde{U}$ is the relative potential between the two bodies. This reduction of the equations of motion is analogous to the reduction of the two-body (two-sphere) problem to a one-body problem.

Equations (12) have precisely the same form as the equations of motion for a material point about an arbitrary general body_-which has been studied extensively for the asteroid problem. ${ }^{3-6}$ One of the main results of these previous studies is that the orbit of the material point can suffer significant changes in its energy and angular momentum as it interacts with the rotating asteroid. Since the general form of these equations does not change, we also expect to find significant changes to the energy and angular momentum of the relative orbit of the general body and sphere-plus the additional expected changes to the rotational dynamics of the general body. ${ }^{7}$

\section{Rotational Equations of Motion}

The rotational dynamics of a sphere are known to be trivial in the presence of a general gravitational force, allowing us to remove it from consideration. The same is not true for the general body, since the gravitational attraction of the sphere will cause non-zero net torques to exist. If the angular momentum of the general body is represented as $\mathbf{H}$ in the body-fixed frame, its angular velocity as $\boldsymbol{\Omega}$, and its inertia tensor as $\mathbf{I}$ the appropriate form of the Euler equations for this interaction is ${ }^{2}$

$$
\dot{\mathbf{H}}=\mathbf{H} \times \boldsymbol{\Omega}+\mathbf{R} \times \frac{\partial V}{\partial \mathbf{R}}
$$




$$
\begin{gathered}
\boldsymbol{\Omega}=\mathbf{I}^{-1} \cdot \mathbf{H} \\
\mathbf{I}=-\int_{\beta_{c}} \tilde{\boldsymbol{\rho}} \cdot \tilde{\rho} d m(\rho),
\end{gathered}
$$

where the equations are specified in the general body-fixed frame, and $\tilde{\rho}$ denotes the cross-product dyad; that is, $\mathbf{a} \times \mathbf{b}=\tilde{a} \cdot \mathbf{b}=\mathbf{a} \cdot \tilde{b}$. The transformation matrix from the body-fixed frame to the inertial frame, $\mathbf{A}$, is then subject to the differential equation

$$
\dot{\mathbf{A}}=\mathbf{A} \times \mathbf{\Omega}
$$

and can be obtained by quadrature following the solution of the translational and rotational equations of motion.

\section{Integrals of Motion}

Both the energy and angular momentum of the entire two-body system (both translational and rotational) are conserved. The presence of these two general integrals (four scalar integrals in all) is where much of the qualitative similarity between the current problem and the problem of a material point orbiting about an asteroid disappear. For the material point problem, we only have conservation of the Jacobi integral (a scalar combination of the energy and angular momentum integrals) if the general body is uniformly rotating ${ }^{5}$ and have no conserved quantities if the general body is in a general rotation state. ${ }^{8}$

For our problem these integrals can be expressed as

$$
\begin{gathered}
E=\frac{1}{2} \dot{\mathbf{r}}_{I} \cdot \dot{\mathbf{r}}_{I}+\frac{1}{2} \boldsymbol{\Omega} \cdot \mathbf{I} \cdot \mathbf{\Omega}-G\left(M_{s}+M_{r}\right) \tilde{U}\left(\mathbf{r}_{I}\right) \\
\mathbf{K}=\mathcal{H}+\frac{M_{c} M_{s}}{M_{c}+M_{s}}\left(\mathbf{r}_{I} \times \dot{\mathbf{r}}_{I}\right),
\end{gathered}
$$

where $E$ is the total energy of the system (minus the rotational energy of the sphere), $\mathbf{K}$ is the total angular momentum of the system (minus the rotational angular momentum of the sphere), and $\mathcal{H}=\mathbf{A} \cdot \mathbf{H}$ is the rotational angular momentum vector of the general body in inertial space.

\section{Normalized Equations of Motion in a Rotating Frame}

Now transform to a coordinate frame fixed to the rotating general body:

$$
\begin{gathered}
\ddot{\mathbf{r}}_{b}+2 \boldsymbol{\Omega} \times \dot{\mathbf{r}}_{b}+\dot{\boldsymbol{\Omega}} \times \mathbf{r}_{b}+\boldsymbol{\Omega} \times\left(\boldsymbol{\Omega} \times \mathbf{r}_{b}\right)=G\left(M_{1}+M_{2}\right) \frac{\partial \tilde{U}}{\partial \mathbf{r}_{b}} \\
\mathbf{I} \cdot \dot{\boldsymbol{\Omega}}+\boldsymbol{\Omega} \times \mathbf{I} \cdot \boldsymbol{\Omega}=-G\left(M_{1}+M_{2}\right) \mathbf{r}_{b} \times \frac{\partial \tilde{U}}{\partial \mathbf{r}_{b}}
\end{gathered}
$$

where $\mathbf{r}_{b}$ is the body-fixed position vector and the time derivatives are assumed to be relative to the rotating frame.

Next, introduce a number of normalizations that simplify our discussion and define the fundamental parameters of our system. These are most easily carried out and defined in the Lagrangian system stated above. To carry out these normalizations, we need to define a fundamental unit of length and a fundamental unit of time. For the length scale we take the maximum radius of the distributed body, denoted by 
$\alpha$. To define the time scale we take the mean motion of the total system at this radius: $n=\sqrt{G\left(M_{1}+M_{2}\right) / \alpha^{3}}$. Define the normalized position vectors by $\mathbf{r}=\mathbf{r}_{b} / \alpha$, the normalized rotational velocity vectors by $\omega=\boldsymbol{\Omega} / n$, and denote normalized time derivatives by ', we find

$$
\begin{gathered}
\mathbf{r}^{\prime \prime}+2 \omega \times \mathbf{r}^{\prime}+\omega^{\prime} \times \mathbf{r}+\omega \times(\omega \times \mathbf{r})=\frac{\partial \mathcal{U}}{\partial \mathbf{r}} \\
I \cdot \omega^{\prime}+\omega \times I \cdot \omega=-v \mathbf{r} \times \frac{\partial \mathcal{U}}{\partial \mathbf{r}} \\
\mathcal{K}=\frac{1}{v} \mathbf{A} \cdot I \cdot \omega+\mathbf{A} \cdot \mathbf{r} \times\left(\mathbf{r}^{\prime}+\omega \times \mathbf{r}\right) \\
\mathcal{E}=\frac{1}{2}\left(\mathbf{r}^{\prime}+\omega \times \mathbf{r}\right) \cdot\left(\mathbf{r}^{\prime}+\omega \times \mathbf{r}\right)+\frac{1}{2 v} \omega \cdot I \cdot \omega-\mathcal{U},
\end{gathered}
$$

where

$$
\begin{gathered}
U=\frac{\alpha}{M_{2}} \int_{\beta_{c}} \frac{d m}{|\mathbf{r}+\rho|} \\
I=-\frac{1}{M_{2} \alpha^{2}} \int_{\beta_{c}} \tilde{\rho} \cdot \tilde{\rho} d m .
\end{gathered}
$$

The free parameter of the system is

$$
v=\frac{M_{1}}{M_{1}+M_{2}},
$$

which is the same parameter as is found in the restricted three body problem. The case when $v \rightarrow 0$ corresponds to the motion of a material point in the gravity field of the distributed body, with main application to orbital dynamics about an asteroid. In this case, we see that the energy and angular momentum integrals are dominated by the rotational dynamics of the distributed body, and that the contribution of the motion of the spherical body decouples from these integrals. The case when $v \rightarrow 1$ corresponds to the motion of a massless distributed body about a point-mass, with application to a large satellite in orbit about a planet. It is important to note that the angular momentum and energy integrals still apply to this problem. There is no singularity when $M_{2} \rightarrow 0$, since the inertia dyad and gravity field of the distributed body are defined relative to the geometry only. This indicates that analysis of gravity gradient satellites should not neglect translational motion, because it is coupled at this fundamental level to the rotational motion.

\section{EQUILIBRIUM SOLUTIONS}

In the above Lagrangian form, the analysis of equilibrium solutions is relatively simple. The conditions for equilibrium (i.e., that all time derivatives be zero) are

$$
\begin{gathered}
\omega \times(\omega \times \mathbf{r})=\frac{\partial \mathcal{U}}{\partial \mathbf{r}} \\
\omega \times I \cdot \omega+\nu \mathbf{r} \times \frac{\partial \mathcal{U}}{\partial \mathbf{r}}=0 .
\end{gathered}
$$


This constitutes a set of six equations for six unknowns, the relative position $\mathbf{r}$ and the angular velocity $\omega$. The solution of these equations is non-trivial, especially for a non-symmetric mass distribution for the gravitational potential. Equation (28) has been solved for arbitrary mass distributions. ${ }^{5,6}$ However, the solution of both equations for "real" gravity fields has not been performed for this class of problems to date. If a symmetry assumption on the gravitational potential is made, however, then solution of these equations is relatively simple, as will be shown later. It is important to note that for $v=0$, the solution to Equation (29) reduces to the classic rigid body rotation results, equilibria are rotations about the principal axes of inertia. To solve these equilibria for general gravity fields with $v \neq 0$, a fruitful approach may be to start from the solutions at $v=0$ and continue them for increasing values of $v$. Such an approach is not carried out here, however.

At equilibrium the integrals of motion reduce to

$$
\begin{gathered}
\mathcal{K}=\frac{1}{\mathrm{v}} \mathbf{A} \cdot I \cdot \omega+\mathbf{A} \cdot \mathbf{r} \times(\omega \times \mathbf{r}) \\
\mathcal{E}=\frac{1}{2}(\omega \times \mathbf{r}) \cdot(\omega \times \mathbf{r})+\frac{1}{2 v} \omega \cdot I \cdot \omega-\mathcal{U} .
\end{gathered}
$$

There are a number of fundamental observations that we can make about properties of the solutions to these equilibrium equations. Although these observations are not new, ${ }^{2}$ we are able to establish them in a concise manner, and thus, repeat them here.

The gravitational acceleration is perpendicular to the spin axis. To show this, we note that the left-hand-side of Equation (28) can be written as follows:

$$
(\omega \cdot \mathbf{r}) \omega-\omega^{2} \mathbf{r}=\mathcal{U}_{r}
$$

and, thus, we immediately see that $\omega \cdot \mathcal{U}_{r}=0$.

The spin vector solves an eigenvector equation. Using the above result for the gravitational acceleration, we insert it into Equation (29) to find

$$
\omega \times I \cdot \omega+(\mathbf{r} \cdot \omega) \mathbf{r} \times \omega=0,
$$

which can be rewritten as

$$
\omega \times[I-v \mathbf{r r}] \cdot \omega=0,
$$

where $\mathbf{r r}$ is a dyad. Assuming that $|\omega| \neq 0$, this means that the expression

$$
[I-v \mathbf{r r}] \cdot \omega
$$

is parallel to the vector $\omega$. Thus, $\omega$ must be an eigenvector of the dyadic $I-\mathbf{r r}$. Note, this does not mean that this dyadic is singular, and in fact it is not, in general.

A formula for the spin rate. Taking the dot product of Equation (32) with the position vector $\mathbf{r}$ yields a simple formula for the spin rate:

$$
\omega^{2}=\frac{-\mathbf{r} \cdot \mathcal{U}_{r}}{r^{2}\left[1-(\hat{\omega} \cdot \hat{\mathbf{r}})^{2}\right]},
$$

where $\hat{\omega}$ and $\hat{\mathbf{r}}$ are the unit vectors along the vectors $\omega$ and $\mathbf{r}$, respectively. Note that this equation is subject to a number of constraints on the position and angular velocity vectors.

The total angular momentum is parallel to $\omega$. To see this, we first rewrite the triple cross product in Equation (30) to find

$$
\mathcal{K}=I \cdot \omega-\operatorname{vr}(\mathbf{r} \cdot \omega)+r^{2} \omega,
$$


but this can be written as

$$
\mathcal{K}=[I-v \mathbf{r r}] \cdot \omega+r^{2} \omega .
$$

The first term is proportional to $\omega$ from the previous paragraph, and thus, the total angular momentum is parallel to $\omega$ and, hence, also perpendicular to $\mathcal{U}_{r}$.

Constraints on the relative position. The position vector lies in the plane defined by the gravitational acceleration and the spin vector. This can be shown by rewriting Equation (32) in the form

$$
\mathbf{r}=\frac{1}{\omega^{2}}(\omega \cdot \mathbf{r}) \omega-\frac{1}{\omega^{2}} U_{r}
$$

From this, we can discover a number of additional constraints.

First, if $\omega$ is along a principal axis of the distributed body, then the eigenvector condition on $\omega$ shows that either $\mathbf{r}$ is parallel to $\omega$ or it is perpendicular to it. If it is parallel to $\omega$, then the gravitational acceleration must be identically zero, from Equation (28), which is not the case. Thus, the position vector must be perpendicular to the spin axis and, hence, parallel to the gravitational acceleration. It is important to note that, for a general mass distribution, the position and gravitational accelerations are not aligned in general. This alignment only occurs for symmetric bodies along certain lines or planes of symmetry. This leads to a complimentary result.

If $\mathbf{r}$ is not parallel to $\mathcal{U}_{r}$, then $\omega$ does not lie along a principal axis of the distributed body. Thus, for general mass distributions; that is, distributions without symmetry; the equilibrium configuration will not rotate about its principal axis.

\section{RELATIVE EQUILIBRIA FOR THE ELLIPSOID-SPHERE SYSTEM}

If we assume that the non-spherical body is a constant density, triaxial ellipsoid the system and conditions for relative equilibria simplify to a large extent. In Reference 4 we find the general equations for the gravitational potential of an ellipsoid applied to an orbital problem (originally derived using Ivory's Theorem) ${ }^{9}$ now stated in our normalized form in a coordinate frame taken along the principal axes of inertia of the ellipsoid:

$$
\begin{gathered}
\mathcal{U}=\frac{3}{4} \int_{\lambda}^{\infty} \phi(\mathbf{r}, u) \frac{d u}{\Delta(u)} \\
\phi(\mathbf{r}, u)=1-\frac{x^{2}}{1+u}-\frac{y^{2}}{\beta^{2}+u}-\frac{z^{2}}{\gamma^{2}+u} \\
\Delta(u)=\sqrt{(1+u)\left(\beta^{2}+u\right)\left(\gamma^{2}+u\right)},
\end{gathered}
$$

where $0<\gamma \leq \beta \leq 1$, and $\lambda$ satisfies the equation $\phi(\mathbf{r}, \lambda)=0$. The $x$-coordinate is along the longest axis of the body (minimum moment of inertia), and the $z$-coordinate is along the shortest axis of the body (maximum moment of inertia); finally, $\beta$ and $\gamma$ are the semiaxes of the ellipsoid divided by the largest axis, $\alpha$, which is the normalizing radius for this problem. The normalized principal moments of inertia for this problem are

$$
I_{x x}=\frac{1}{5}\left(\beta^{2}+\gamma^{2}\right)
$$




$$
\begin{aligned}
& I_{y y}=\frac{1}{5}\left(1+\gamma^{2}\right) \\
& I_{z z}=\frac{1}{5}\left(1+\beta^{2}\right) .
\end{aligned}
$$

With this form of the force potential the first and second partials of $\mathcal{U}$ are easily computed

$$
\begin{aligned}
& u_{x}=-\frac{3}{2} x \int_{\lambda}^{\infty} \frac{d u}{(1+u) \Delta(u)} \\
& \mathcal{U}_{y}=-\frac{3}{2} y \int_{\lambda}^{\infty} \frac{d u}{\left(\beta^{2}+u\right) \Delta(u)} \\
& \mathcal{U}_{z}=-\frac{3}{2} x \int_{\lambda}^{\infty} \frac{d u}{\left(\gamma^{2}+u\right) \Delta(u)} \\
& \mathcal{U}_{x x}=-\frac{3}{2} \int_{\lambda}^{\infty} \frac{d u}{(1+u) \Delta(u)}+\frac{3 x^{2}}{(1+\lambda)^{2} \Delta(\lambda)} \frac{1}{\left[\frac{x^{2}}{(1+\lambda)^{2}}+\frac{y^{2}}{\left(\beta^{2}+\lambda\right)^{2}}+\frac{z^{2}}{\left(\gamma^{2}+\lambda\right)^{2}}\right]} \\
& U_{y y}=-\frac{3}{2} \int_{\lambda}^{\infty} \frac{d u}{\left(\beta^{2}+u\right) \Delta(u)}+\frac{3 y^{2}}{\left(\beta^{2}+\lambda\right)^{2} \Delta(\lambda)} \frac{1}{\left[\frac{x^{2}}{(1+\lambda)^{2}}+\frac{y^{2}}{\left(\beta^{2}+\lambda\right)^{2}}+\frac{z^{2}}{\left(\gamma^{2}+\lambda\right)^{2}}\right]} \\
& \mathcal{U}_{z z}=-\frac{3}{2} \int_{\lambda}^{\infty} \frac{d u}{\left(\gamma^{2}+u\right) \Delta(u)}+\frac{3 y^{2}}{\left(\gamma^{2}+\lambda\right)^{2} \Delta(\lambda)} \frac{1}{\left[\frac{x^{2}}{(1+\lambda)^{2}}+\frac{y^{2}}{\left(\beta^{2}+\lambda\right)^{2}}+\frac{z^{2}}{\left(\gamma^{2}+\lambda\right)^{2}}\right]} \\
& u_{x y}=\frac{3 x y}{\Delta(\lambda)(1+\lambda)\left(\beta^{2}+\lambda\right)} \frac{1}{\left[\frac{x^{2}}{(1+\lambda)^{2}}+\frac{y^{2}}{\left(\beta^{2}+\lambda\right)^{2}}+\frac{z^{2}}{\left(\gamma^{2}+\lambda\right)^{2}}\right]} \\
& \mathcal{U}_{y z}=\frac{3 y z}{\Delta(\lambda)\left(\beta^{2}+\lambda\right)\left(\gamma^{2}+\lambda\right)} \frac{1}{\left[\frac{x^{2}}{(1+\lambda)^{2}}+\frac{y^{2}}{\left(\beta^{2}+\lambda\right)^{2}}+\frac{z^{2}}{\left(\gamma^{2}+\lambda\right)^{2}}\right]} \\
& \mathcal{U}_{z x}=\frac{3 z x}{\Delta(\lambda)\left(\gamma^{2}+\lambda\right)(1+\lambda)} \frac{1}{\left[\frac{x^{2}}{(1+\lambda)^{2}}+\frac{y^{2}}{\left(\beta^{2}+\lambda\right)^{2}}+\frac{z^{2}}{\left(\gamma^{2}+\lambda\right)^{2}}\right]}
\end{aligned}
$$

We immediately find that relative equilibria exist when the sphere is placed along any of the principal axes of the ellipsoid. Since the position and acceleration vectors are parallel at any of these points, we find that the ellipsoid will spin about one of its other principal axes. Thus, for these solutions, the spin vector is parallel to the axis of inertia and perpendicular to the position vector. Applying our simple formula for the spin rate, we find that, given a position along the $q$-axis, the spin rate must be

$$
\omega^{2}=\frac{3}{2} \int_{\lambda}^{\infty} \frac{d u}{\left(\alpha_{q}^{2}+u\right) \Delta(u)},
$$


where $\lambda=q^{2}-\alpha_{q}^{2}$ and $q$ can equal either $x, y$, or $z$ and $\alpha_{q}$ is equal to $1, \beta$, or $\gamma$, respectively. We note that the spin rate is independent of which principal axis the ellipsoid rotates about.

Using these expressions, the second partials then simplify to

$$
\begin{gathered}
\mathcal{U}_{r s}=0, \quad \forall r \neq s \\
\mathcal{U}_{q q}=-\omega^{2}+\frac{3}{\Delta(\lambda)} \\
\mathcal{U}_{r r}=-\frac{3}{2} \int_{\lambda}^{\infty} \frac{d u}{\left(\alpha_{r}^{2}+u\right) \Delta(u)}, \quad r \neq q,
\end{gathered}
$$

where $r$ and $s$ denote axes other than $q$ unless otherwise indicated, and $\alpha_{r}$ and $\alpha_{s}$ denote the semimajor axes along these directions.

We note the following identity:

$$
\frac{1}{\Delta(\lambda)}=\frac{1}{2} \int_{\lambda}^{\infty}\left[\frac{1}{\alpha_{q}^{2}+u}+\frac{1}{\alpha_{r}^{2}+u}+\frac{1}{\alpha_{s}^{2}+u}\right] \frac{d u}{\Delta(u)},
$$

where $q, r$, and $s$ are distinct. This can be easily proved by taking the derivative of both sides with respect to $\lambda$. Using this result, we establish a number of items that are useful for our later discussion. First we note that

$$
\begin{aligned}
\mathcal{U}_{q q} & =\frac{3}{\Delta(\lambda)}-\frac{3}{2} \int_{\lambda}^{\infty} \frac{d u}{\left(\alpha_{q}^{2}+u\right) \Delta(u)} \\
& =\frac{3}{2} \int_{\lambda}^{\infty}\left[\frac{1}{\alpha_{r}^{2}+u}+\frac{1}{\alpha_{s}^{2}+u}\right] \frac{d u}{\Delta(u)},
\end{aligned}
$$

which clearly shows that $\mathcal{U}_{q q}>0$. We also consider two additional situations, encountered later,

$$
\begin{gathered}
3 \omega^{2}-\mathcal{U}_{r r}-\frac{3}{\Delta(\lambda)}=\frac{3}{2} \int_{\lambda}^{\infty}\left[\frac{2}{\alpha_{q}^{2}+u}-\frac{1}{\alpha_{s}^{2}+u}\right] \frac{d u}{\Delta(u)} \\
4 \omega^{2}-\frac{3}{\Delta(\lambda)}=\frac{3}{2} \int_{\lambda}^{\infty}\left[\frac{3}{\alpha_{q}^{2}+u}-\frac{1}{\alpha_{r}^{2}+u}-\frac{1}{\alpha_{s}^{2}+u}\right] \frac{d u}{\Delta(u)} .
\end{gathered}
$$

We cannot find definitive inequalities for these combinations, indeed under certain circumstances they can take on positive or negative values. We can establish the following results, however. First, if $\alpha_{q}<\alpha_{s}$ then Equation (62) is always positive, and if $\alpha_{q}<\alpha_{r}$ as well, then Equation (63) is also always positive. If $\alpha_{q}>\alpha_{s}$ then the sign of Equation (62) is not definite in general, but a sufficient condition for it to be positive is that $q^{2}>2\left(\alpha_{q}^{2}-\alpha_{s}^{2}\right)$. If $\alpha_{q} \leq \alpha_{r}$ then the same sufficient condition will guarantee that (63) is positive. If $\alpha_{q}$ is greater than both $\alpha_{r}$ and $\alpha_{s}$, a sufficient condition for (63) to be positive is that $q^{2} \gg 2\left[2 \alpha_{q}^{2}-\alpha_{r}^{2}-\alpha_{s}^{2}\right]$.

Finally, consider the quantity

$$
\omega^{2}+\mathcal{U}_{r r}=\frac{3}{2} \int_{\lambda}^{\infty}\left[\frac{1}{\alpha_{q}^{2}+u}-\frac{1}{\alpha_{r}^{2}+u}\right] \frac{d u}{\Delta(u)} .
$$

It is simple to note that (64) is positive if $\alpha_{q}<\alpha_{r}$ and negative if $\alpha_{q}>\alpha_{r}$. 


\section{STABILITY}

Knowing that these equilibrium solutions exist, we now want to determine their stability. In this paper, we consider the simpler case of in-plane stability, leaving the more general (and difficult) case of out-of-plane instability for a later paper. Due to the symmetry of the ellipsoidal model, we can restrict ourselves to motion in a plane. The main advantage of this restriction is that the angular momentum can be eliminated and the system can be reduced to a two-degree of freedom Hamiltonian system. The advantage of expressing this system as a Hamiltonian system is clear, since the stability properties of these systems contain many symmetries and are easy to evaluate.

The steps from the above Lagrangian set of equations to a Hamiltonian formulation are relatively simple once we restrict ourselves to planar motion. First, we define the rotation pole of the ellipsoid and the direction of the total angular momentum to be aligned along one of the principal axes of the system, denoted by $\hat{z}$. Then, the coordinates of the position vector in the plane perpendicular to $\hat{z}$ are denoted by $\mathbf{q}=\mathbf{r}$. Next, we define the momenta to be the inertial velocity, $\mathbf{p}=\mathbf{r}^{\prime}+\omega \times \mathbf{r}$. The energy and angular momentum integrals can be recast as

$$
\begin{gathered}
\mathcal{E}=\frac{1}{2} \mathbf{p} \cdot \mathbf{p}+\frac{1}{2 v} \omega \cdot I \cdot \omega-\mathcal{U}(\mathbf{q}) \\
\mathcal{K} \hat{\mathbf{z}}=\frac{1}{v} I_{z z} \omega \hat{\mathbf{z}}+\mathbf{q} \times \mathbf{p} .
\end{gathered}
$$

Now, the time rate of change of $\mathbf{q}$ and $\mathbf{p}$ are just

$$
\begin{gathered}
\mathbf{p}^{\prime}+\omega \times \mathbf{p}=U_{\mathbf{q}} \\
\mathbf{q}^{\prime}+\omega \times \mathbf{q}=\mathbf{p}
\end{gathered}
$$

and we still, in general, must solve for the angular velocity from our equation for $\omega^{\prime}$.

Given our constraint for planar motion, the angular velocity can be solved uniquely from the angular momentum integral

$$
\omega=\frac{v}{I_{z z}} \cdot[\mathcal{K}-\hat{\mathbf{z}} \cdot(\mathbf{q} \times \mathbf{p})] .
$$

Substituting this into the energy integral and introducing the notation $\mathcal{E}=\mathcal{H}$ yields:

$$
\mathcal{H}=\frac{1}{2} \mathbf{p} \cdot \mathbf{p}+\frac{v}{2 I_{z z}}(\mathcal{K}-\hat{\mathbf{z}} \cdot(\mathbf{q} \times \mathbf{p}))^{2}-\mathcal{U}(\mathbf{q}),
$$

where we note that

$$
\begin{aligned}
\mathbf{q}^{\prime} & =\mathcal{H}_{\mathbf{p}} \\
\mathbf{p}^{\prime} & =-\mathcal{H}_{\mathbf{q}} .
\end{aligned}
$$

It is easy to show that this Hamiltonian is a constant and that it generates the equations of motion. The angular momentum is now a parameter that must be specified. Once the solution is generated, the angular velocity is directly solved from the angular momentum integral.

In a principal axis frame the equilibrium solution is found to be $q_{1}=q, q_{2}=0$, $p_{1}=0, p_{2}=\omega q, \omega$ defined by (55). Given an equilibrium solution for the system, its linear stability can now be determined from the characteristic equation 


$$
A=\left[\begin{array}{cccc}
|\xi I-A|= & 0 \\
0 & \omega & 1 & 0 \\
\omega(\sigma-1) & 0 & 0 & 1+\sigma \\
\frac{3}{\Delta(\lambda)}-(1+\sigma) \omega^{2} & 0 & 0 & \omega(1-\sigma) \\
0 & U_{r r}-\omega & 0
\end{array}\right]
$$

where $\xi$ is the eigenvalue. Due to the symmetries of a Hamiltonian system the resulting characteristic equation has the form

$$
\xi^{4}+a \xi^{2}+b=0,
$$

where

$$
\begin{gathered}
a=3 \omega^{2}-\mathcal{U}_{r r}-\frac{3}{\Delta(\lambda)}-\sigma\left(\omega^{2}+\mathcal{U}_{r r}\right) \\
b=\left(\omega^{2}+\mathcal{U}_{r r}\right)\left[\frac{3}{\Delta(\lambda)}-\sigma\left(4 \omega^{2}-\frac{3}{\Delta(\lambda)}\right)\right] \\
\lambda=q^{2}-\alpha_{q}^{2} .
\end{gathered}
$$

Given this system, the stability conditions are easily evaluated,

$$
\begin{gathered}
a>0 \\
b>0 \\
a^{2}-4 b>0 .
\end{gathered}
$$

In general, one should consider all three basic cases, equilibrium along the long axis of the body, or $\alpha_{q} \geq \alpha_{r}$, equilibrium along the intermediate axis of the body, $\alpha_{s} \leq \alpha_{q} \leq \alpha_{r}$, or equilibrium along the minimum axis of the body, $\alpha_{q} \leq \alpha_{s} \leq \alpha_{r}$. For each of these solutions the body can rotate about either of the other two principal axes, generating a total of six distinct cases.

For the remainder of the discussion we assume that the ellipsoid rotates about its maximum moment of inertia, disregarding the other possible situations that may exist. This is largely motivated by natural systems, which energetically favor rotation about their maximum moment of inertia and in general do not rotate about their other principal axes. When we consider the full non-planar stability of these equations we will reconsider this case, however. See FiguRE 1 for a graphic depictions of the two cases that can occur for this situation. For each of these cases, we can establish a few inequalities on some of the terms in (80) and (81). Namely, for $\sigma=0$, the coefficient $b<0$ for the long-axis equilibrium and $b>0$ for the short-axis equilibrium. For the motion of a massless particle about an ellipsoid, we find that the long-axis solutions are always unstable but that the short axis solutions may be stable. ${ }^{4}$

This is interesting, because if we take the alternate case, $v=1$ and $q \gg 1$ we know, from an extensive literature, ${ }^{10}$ that the long-axis solution is stable and corresponds to the well-known gravity-gradient satellite configuration. Also, we know that in these cases the short-axis solutions are always unstable. From the limits and inequalities 

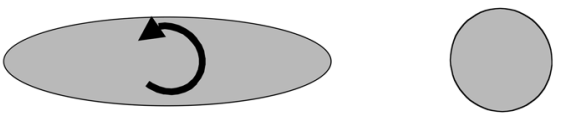

Long-Axis Equilibria

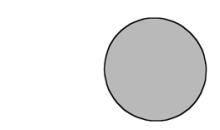

Short-Axis Equilibria

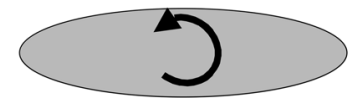

FIGURE 1. Long and short axis relative equilibrium configurations.

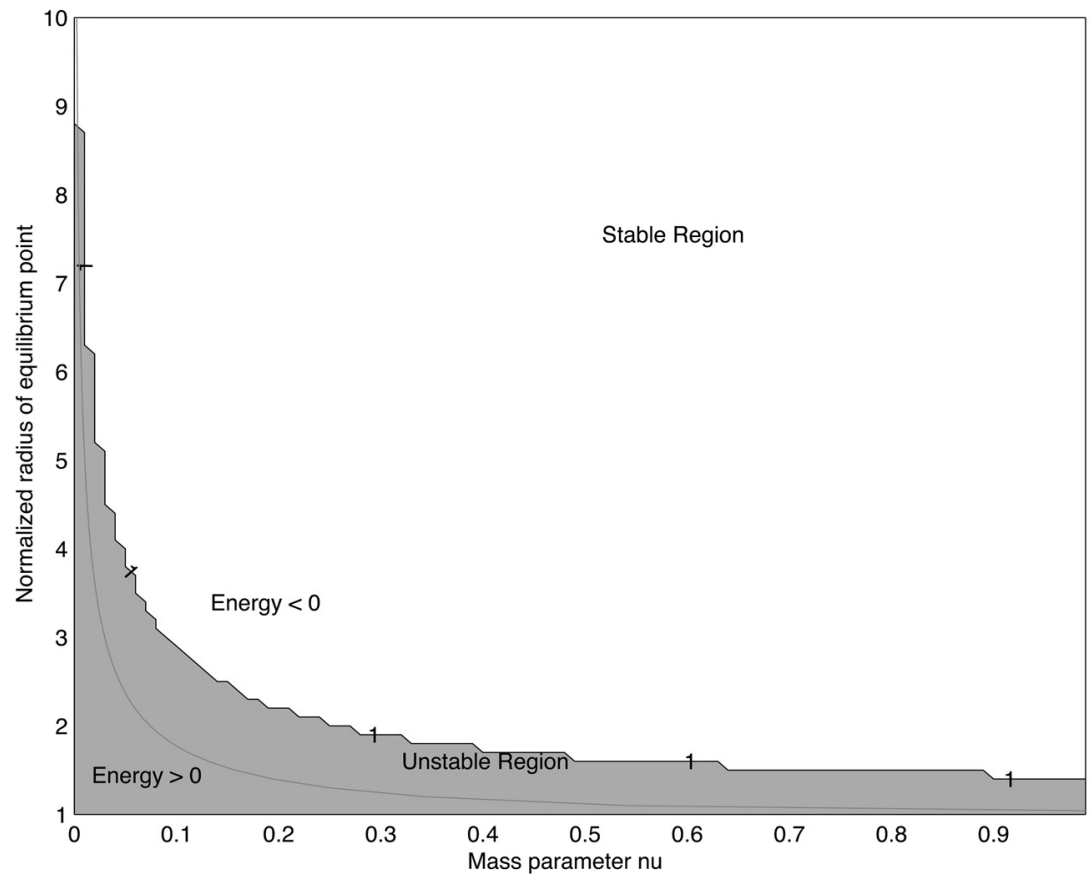

FIGURE 2. Stability diagram for planar motion in the long-axis solution. White denotes spectral stability and gray denotes a single hyperbolic manifold instability. 
we noted previously, we see that if $\sigma$ is sufficiently large (i.e., if $q$ is large enough) the long-axis solution should satisfy $a$ and $b$ positive, and by computation we find that (82) is also satisfied. Conversely, the short-axis solution in this situation will always have both $a$ and $b$ negative for large enough $q$. Thus, in this more general case we see that the standard results for gravity-gradient satellites may have to be revised for the more general case when both bodies have mass. Results for an ellipsoid with semimajor axes of 1:0.5:0.5 are shown in FIGURES 2 and 3. On these plots we have also shown the line where the total energy of the system changes from positive to negative. This is significant, since a system with negative total energy cannot evolve into an escaping system, whereas a system with positive energy can, if sufficiently perturbed from its relative equilibrium. ${ }^{11}$

\section{CONCLUSIONS}

In this paper we define the full two-body problem for the interaction of a sphere and an ellipsoid. We discuss the relative equilibria for the general problem, not assuming any additional symmetries beyond the standard triaxial ellipsoid model.

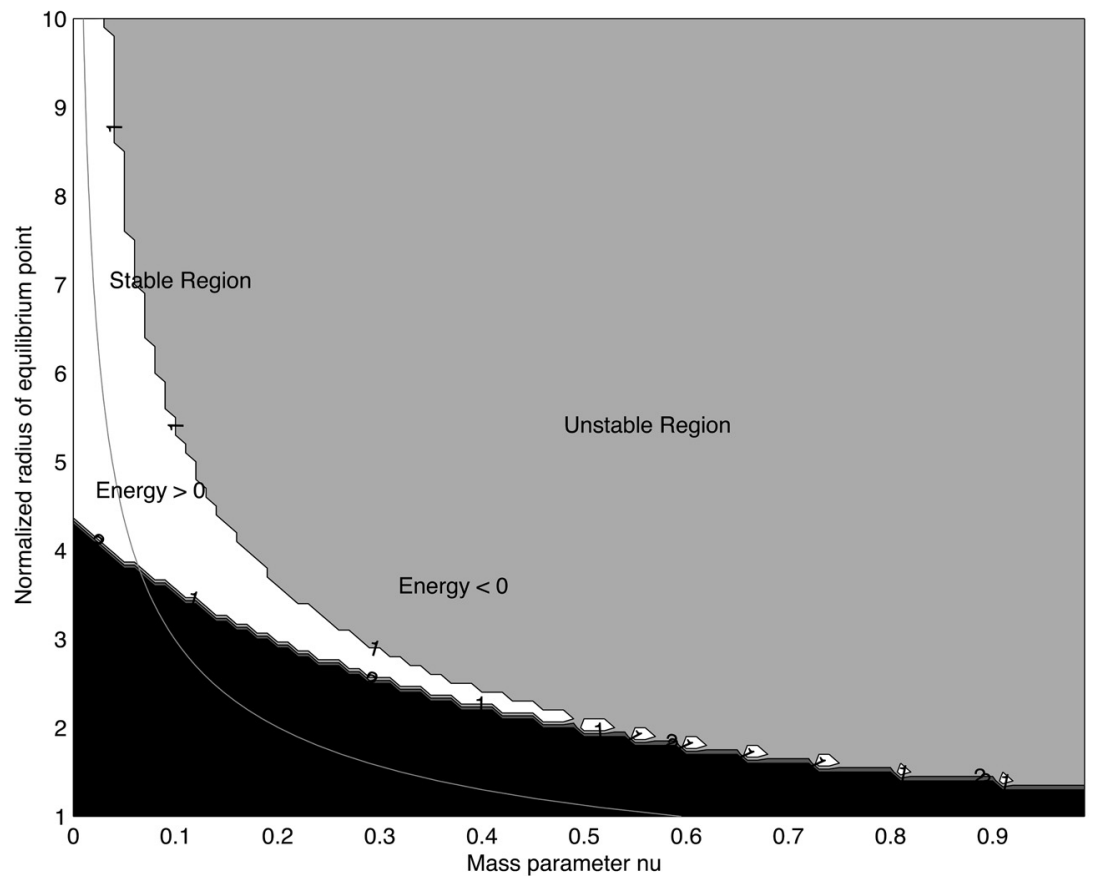

FIGURE 3. Stability diagram for planar motion in the short-axis solution. White denotes spectral stability, gray denotes a single hyperbolic manifold instability, and black denotes spiral manifold instability. 
We examine the spectral stability for planar motion in this system, reserving a discussion of non-planar stability for a future analysis.

\section{ACKNOWLEDGMENTS}

This work was supported by grants to the University of Michigan from NASA's Planetary Geology and Geophysics program and by the Jet Propulsion Laboratory's IND Technology Program.

\section{REFERENCES}

1. GoŹDziewski, K. \& A.J. Maciejewski. 1999. Unrestricted planar problem of a symmetric body and a point mass. Triangular libration points and their stability. Celest. Mech. Dyn. Astron. 75: 251-285.

2. MACIEJEWSKI, A.J. 1995. Reduction, relative equilibria and potential in the two rigid bodies problem. Celest. Mech. Dyn. Astron. 63: 1-28.

3. Chauvineau, B., P. Farinella \& F. Mignard. 1993. Planar orbits about a triaxial body: applications to asteroidal satellites. Icarus 105: 370-384.

4. ScheERES, D.J. 1994. Dynamics about uniformly rotating tri-axial ellipsoids. Applications to asteroids. Icarus 110: 225-238.

5. Scheeres D.J., S.J. Ostro, R.S. Hudson \& R.A. Werner. 1996. Orbits close to asteroid 4769 Castalia. Icarus 121: 67-87.

6. Scheeres, D.J., B.G. Williams \& J.K. Miller. 2000. Evaluation of the dynamic environment of an asteroid: applications to 433 Eros. J. Guidance Control Dynam. 23: 466-475.

7. SCHEERES, D.J. 2002. Stability of binary asteroids. Icarus 159: 271-283.

8. ScheERES, D.J., S.J. Ostro, R.S. HudSON, et al. 1998. Dynamics of orbits close to asteroid 4179 Toutatis. Icarus 132: $53-79$.

9. Danby, J.M.A. 1992. Fundamentals of Celestial Mechanics, 2nd edit. Willmann-Bell, Richmond.

10. BECK, J.A. \& C.D. HALl. 1998. Relative equilibria of a rigid satellite in a circular keplerian orbit. J. Astronaut. Sci. 46: 215-247.

11. ScheEres, D.J. 2002. Stability in the full two-body problem. Celest. Mech. Dyn. Astron. 83: 155-169. 